\title{
Arterial stiffness is associated with target organ damage in subjects with pre-hypertension
}

Shaoyun $\mathrm{Wu}^{1}$, Dubo Chen ${ }^{2}$, Xun Zeng ${ }^{3}$, Junmin Wen ${ }^{4}$, Chuzhi Zhou ${ }^{4}$, Ke Xiao ${ }^{5}$, Peng Hu ${ }^{6}$, Weixin Chen ${ }^{4}$

${ }^{1}$ Department of Cardiology, the First Affiliated Hospital of Sun Yat-sen University, Guangzhou, China

2Department of Laboratory Medicine, the First Affiliated Hospital of Sun Yat-sen University, Guangzhou, China

${ }^{3}$ Department of Outpatient, the First Affiliated Hospital of Sun Yat-sen University, Guangzhou, China

${ }^{4}$ Department of Intensive Unit, Shenzhen Sun Yat-sen Cardiovascular Hospital, Shenzhen, China

${ }^{5}$ Department of Cardiology, Shenzhen Sun Yat-sen Cardiovascular Hospital, Shenzhen, China

${ }^{6}$ Department of Cardiology, the Fifth subsidiary Sun Yat-sen University Hospital, Zhuhai, China

Submitted: 17 June 2017

Accepted: 1 July 2017

Arch Med Sci 2018; 14, 6: 1374-1380

DOI: https://doi.org/10.5114/aoms.2017.69240

Copyright $\odot 2017$ Termedia \& Banach

\section{Abstract}

Introduction: Present study was to evaluate whether increased arterial stiffness was associated with target organ damage in pre-hypertensive subjects. Material and methods: Pre-hypertensive subjects enrolled and echocardiography was used to evaluate left ventricular hypertrophy (LVH) and the first morning urine was collected to evaluate albumin and creatinine ratio (ALB/ $\mathrm{Cr}$ ratio). Carotid-femoral pulse wave velocity (cf-PWV) was measured.

Results: A total of 420 subjects were recruited and mean age was 42.6 years. Mean systolic/diastolic blood pressure (SBP/DBP) were $130 \pm 9 \mathrm{~mm} \mathrm{Hg}$ and $85 \pm 4 \mathrm{~mm} \mathrm{Hg}$. The prevalence of albuminuria and left ventricular hypertrophy was $8.6 \%$ and $11.7 \%$. Mean cf-PWV was $9.2 \pm 1.0 \mathrm{~m} / \mathrm{s}$, with arterial stiffness prevalence was $8.8 \%$. Subjects with arterial stiffness had higher cf-PWV value $(10.6 \pm 0.4 \mathrm{~m} / \mathrm{s}$ vs. $8.7 \pm 0.6 \mathrm{~m} / \mathrm{s}, p<0.05)$, and $\mathrm{ALB} / \mathrm{Cr}$ ratio $(28.3 \pm 13.2 \mu \mathrm{g} / \mathrm{mg}$ vs. $23.1 \pm 11.4 \mu \mathrm{g} / \mathrm{mg}, p<0.05)$. Overall, with multivariate regression analysis, aging and arterial stiffness were significantly associated with pre-hypertension. With stepwise adjusted for potential covariates including age, male gender, fasting plasma glucose, presence of current cigarette smoking, dyslipidemia, statins and SBP, increased cf-PWV remained independently associated with left ventricular hypertrophy and albuminuria, with an increased odds of $41 \%$ and $24 \%(p<0.05)$, respectively.

Conclusions: In pre-hypertensive subjects, arterial stiffness is independently associated with LVH and albuminuria and cf-PWV may be a useful marker to identify target organ damage in pre-hypertensive subjects.

Key words: pre-hypertension, target organ damage, arterial stiffness.

\section{Introduction}

Hypertension is a well-known risk factor for a variety of cardiovascular and renal diseases [1-3]. Nowadays, it is estimated that more than

\author{
Corresponding authors: \\ Peng Hu \\ Department of Cardiology \\ The Fifth Subsidiary \\ Sun Yat-Sen University \\ Hospital \\ Zhuhai 51000 , China \\ Phone: +86 756-2528888 \\ Fax: +86 756-2528888 \\ E-mail: pengh5@mail.sysu. \\ edu.cn
}

Weixin Chen

Department of Intensive Unit

Shenzhen Sun

Yat-sen Cardiovascular

Hospital, Shzhen, China

E-mail: cxw526@126.com 
1 billion people have hypertension around the world $[4,5]$. Furthermore, the prevalence of pre-hypertension, which is defined by a systolic blood pressure (SBP) from 120 to $139 \mathrm{~mm} \mathrm{Hg}$ or a diastolic BP (DBP) from 80 to $89 \mathrm{~mm} \mathrm{Hg}$ [6], has also been dramatically increasing in recent decades. The Prospective Studies Collaboration [7], which included data from 61 observational studies, shows that for every $20 / 10 \mathrm{~mm} \mathrm{Hg}$ increase in SBP and DBP, the risk of cardiovascular disease and mortality is increased two-fold, and this relationship extends to a BP level of $115 / 75 \mathrm{~mm} \mathrm{Hg}$. These data together imply that treatment of pre-hypertension should be beneficial for reducing target organ damage and cardiovascular events.

Arterial stiffness is a pathophysiological process of vascular ageing, and prior observational studies suggest that arterial stiffness is highly prevalent in subjects with hypertension [8-11]. Nevertheless, the prevalence of arterial stiffness in subjects with pre-hypertension is unclear, and whether arterial stiffness is associated with target organ damage in subjects with pre-hypertension is also less well studied.

Using data from a cross-sectional study, we evaluated the prevalence of arterial stiffness in subjects with pre-hypertension and potential risk factors for pre-hypertension. Moreover, whether arterial stiffness was independently associated with the prevalence of target organ damage including left ventricular hypertrophy and albuminuria in pre-hypertensive subjects was also evaluated.

\section{Material and methods}

\section{Studied participants}

The current study was approved by the Research Ethic Committee of the Fifth subsidiary Hospital of Sun Yat-sen University, and all studied participants were provided informed consent before recruitment. The current study was conducted during January of 2015 to December of 2016. Inclusion criteria were as follows: 2 separated times of diagnosis of pre-hypertension in the outpatient clinic, and without a history of previous treatment with BP-lowering drugs. Exclusion criteria were as follows: age younger than 18 years or older than 75 years; with documented cardiovascular diseases including myocardial infarction, cerebrovascular diseases, peripheral artery disease, congestive heart failure, hypertrophic cardiomyopathy, chronic kidney disease and documented type 1 or 2 diabetes mellitus. Demographic data including age, gender, current smoking status, medical history and current medication usage were collected by two investigators using a structured questionnaire.

\section{Diagnosis of pre-hypertension}

Subjects whose clinic BP was consistent with the diagnosis of pre-hypertension during the first outpatient visit were asked to take a second evaluation of clinic BP on another day, and those diagnosed with pre-hypertension at the second visit were asked to participate in the current study and informed consent was provided. In brief, clinic BP measurement was performed in accordance with the JNC7 guideline recommendation [12]. Patients sat quietly for 10 min with their back supported before BP measurements and arms were placed at the level of the heart. The non-dominant arm was used to measure clinic BP and 3 readings were obtained with a 1 min interval between readings. The physician was required to be out of the clinic office during BP measurement. The average value of clinic BP was obtained by calculating the last two $\mathrm{BP}$ readings.

\section{Biochemical indices}

Subjects who agreed to participate in the current study were asked to come back to the outpatient clinic for measurement of biochemical indices. At least $8 \mathrm{~h}$ overnight fasting was required and venous blood was drawn for lipid profiles, fasting plasma glucose and creatinine evaluation. The first urine in the morning was collected to evaluate the albumin $(\mu \mathrm{g} / \mathrm{l})$ and creatinine $(\mathrm{mg} / \mathrm{l})$ ratio. Accordingly [13], a ratio lower than $30 \mu \mathrm{g} / \mathrm{mg}$ is considered as normal, $30-300 \mu \mathrm{g} / \mathrm{mg}$ is microalbuminuria and higher than $300 \mu \mathrm{g} / \mathrm{mg}$ is macroalbuminuria.

\section{Arterial stiffness evaluation}

Carotid-femoral pulse wave velocity (cf-PWV) was used to evaluate arterial stiffness on the same day after drawing blood. Applanation tonometry was used to measure cf-PWV (SphygmoCor; AtCor Medical, Sydney, Australia). Briefly, the distance traveled by the pulse wave over the surface of the body was measured with a tape measure from the sternal notch to the right carotid artery, and from the sternal notch to the right femoral artery. The time delay was measured between the troughs of the two waveforms. Then, the distance was divided by the transit time. Measurement was performed between 8-10 am. Subjects were required to fast and lie in a supine position after resting for $10 \mathrm{~min}$. Three measurements were obtained to obtain the median based on the guideline recommendation [14].

\section{Echocardiography}

The left ventricular posterior wall (LVPW) thickness and interventricular septal (IVS) thickness 


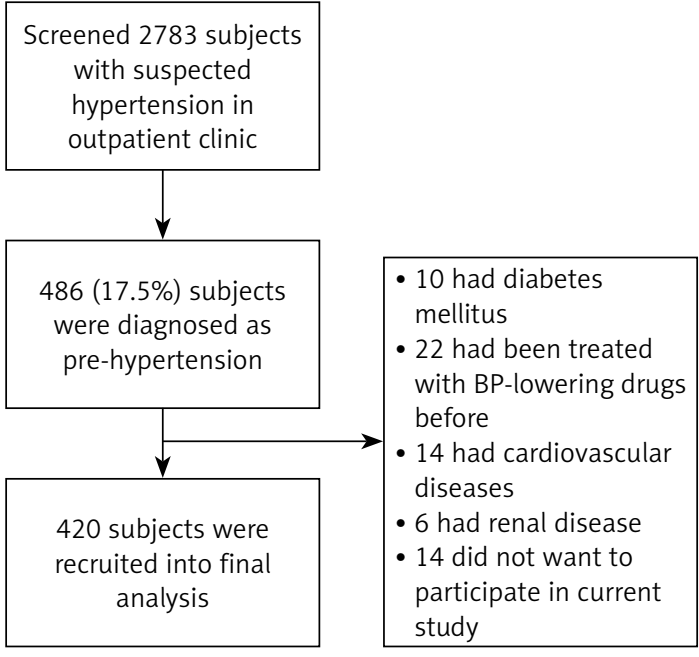

Figure 1. Schematic of study design

Table I. General characteristics

\begin{tabular}{|c|c|}
\hline Variables & Value \\
\hline$N$ & 420 \\
\hline Age [years] & $42.6 \pm 13.5$ \\
\hline Male, $n(\%)$ & $276(65.7)$ \\
\hline Current smoking, $n(\%)$ & $117(27.9)$ \\
\hline $\mathrm{SBP}[\mathrm{mm} \mathrm{Hg}]$ & $130 \pm 9$ \\
\hline $\mathrm{DBP}[\mathrm{mm} \mathrm{Hg}]$ & $85 \pm 4$ \\
\hline Heart rate $[\mathrm{bpm}]$ & $76 \pm 12$ \\
\hline Total cholesterol [mmol/l] & $4.9 \pm 1.1$ \\
\hline Triglyceride [mmol/l] & $1.8 \pm 0.6$ \\
\hline LDL-C $[\mathrm{mmol} / \mathrm{I}]$ & $3.1 \pm 0.7$ \\
\hline $\mathrm{HDL}-\mathrm{C}[\mathrm{mmol} / \mathrm{l}]$ & $1.3 \pm 0.4$ \\
\hline $\mathrm{FPG}[\mathrm{mmol} / \mathrm{l}]$ & $5.9 \pm 0.4$ \\
\hline Creatinine $[\mu \mathrm{mol} / \mathrm{l}]$ & $73.3 \pm 14.2$ \\
\hline $\mathrm{ALB} / \mathrm{Cr}$ ratio $[\mu \mathrm{g} / \mathrm{mg}]$ & $26.7 \pm 11.5$ \\
\hline IVS [mm] & $10.4 \pm 1.9$ \\
\hline LVPW $[\mathrm{mm}]$ & $10.5 \pm 1.7$ \\
\hline Left ventricular hypertrophy, $n$ (\%) & $49(11.7)$ \\
\hline Albuminuria, $n$ (\%) & $36(8.6)$ \\
\hline Dyslipidemia, $n$ (\%) & $52(12.4)$ \\
\hline Aspirin, $n(\%)$ & $33(7.9)$ \\
\hline Statins, $n(\%)$ & $45(10.7)$ \\
\hline $\mathrm{cf}-\mathrm{PWV}[\mathrm{m} / \mathrm{s}]$ & $9.2 \pm 1.0$ \\
\hline Arterial stiffness, $n(\%)$ & $37(8.8)$ \\
\hline
\end{tabular}

$S B P$ - systolic blood pressure, DBP - diastolic blood pressure, $L D L-C$ low-density lipoprotein cholesterol, HDL-C - high-density lipoprotein cholesterol, FPG - fasting plasma glucose, $\mathrm{ALB} / \mathrm{Cr}$ - albumin/ creatinine, IVS - interventricular septal, LVPW - left ventricular posterior wall, cf-PWV - carotid-femoral pulse wave velocity. were measured by echocardiography at the left lower sternal border with the long-axis view. Accordingly [15], the thickness of both the LVPW and IVS greater than or equal to $12 \mathrm{~mm}$ was considered as left ventricular hypertrophy.

\section{Statistical analysis}

Continuous variables were presented as mean \pm SD and categorical variables were presented as number and percentages of cases. Student's $t$-test was used for comparison of continuous variables and the $\chi^{2}$ or Fisher exact test was used for comparison of categorical variables. Logistic regression analysis was used to evaluate the potential risk factors for pre-hypertension; in addition, the association between per 1-SD standardized increase in cf-PWV and prevalence of left ventricular hypertrophy and albuminuria were also evaluated. Statistical analyses were performed using SPSS 17.0 (SPSS Inc, Chicago, IL). All statistical tests were two-sided and considered statistically significant when $p<0.05$.

\section{Results}

\section{General characteristics}

From January 2015 to December 2016, we screened 2783 subjects with suspected hypertension in the outpatient clinic; a total of $486(17.5 \%)$ subjects with pre-hypertension were identified and 420 subjects were recruited into the final analysis. The study flow chart is presented in Figure 1. As shown in Table I, the mean age was 42.6 years, male subjects accounted for $65.7 \%$, and $27.9 \%$ were current cigarette smokers. The mean SBP and DBP were $130 \pm 9 \mathrm{~mm} \mathrm{Hg}$ and $85 \pm 4$ $\mathrm{mm} \mathrm{Hg}$, respectively. The mean $\mathrm{ALB} / \mathrm{Cr}$ ratio was $26.7 \pm 11.5 \mu \mathrm{g} / \mathrm{mg}$, prevalence of albuminuria was $8.6 \%$, the mean IVS and LVPW were $10.4 \pm 1.9 \mathrm{~mm}$ and $10.5 \pm 1.7 \mathrm{~mm}$, respectively, and prevalence of left ventricular hypertrophy was $11.7 \%$. The mean cf-PWV was $9.2 \pm 1.0 \mathrm{~m} / \mathrm{s}$, and prevalence of arterial stiffness was $8.8 \%$ based on the recommended diagnostic criteria [14].

\section{Comparisons between subjects with and without arterial stiffness}

Subjects were divided into two groups and between-group differences were evaluated. As presented in Table II, compared to subjects without arterial stiffness, those with arterial stiffness had a higher cf-PWV value (10.6 \pm 0.4 $\mathrm{m} / \mathrm{s}$ vs. $8.7 \pm 0.6 \mathrm{~m} / \mathrm{s})$. In addition, subjects with arterial stiffness were more likely to be cigarette smokers, and had higher prevalence of left ventricular hypertrophy and albuminuria $(p<0.05$ for all comparisons). 
Table II. Comparisons between subjects with and without arterial stiffness

\begin{tabular}{|c|c|c|}
\hline Variables & With arterial stiffness & Without arterial stiffness \\
\hline N & 37 & 383 \\
\hline Age [years] & $44.3 \pm 12.7$ & $41.7 \pm 13.8$ \\
\hline Male, $n(\%)$ & $24(64.9)$ & $252(65.8)$ \\
\hline Current smoking, $n$ (\%) & $12(44.4)^{\star}$ & $105(31.1)$ \\
\hline $\mathrm{SBP}[\mathrm{mm} \mathrm{Hg}]$ & $132 \pm 7$ & $129 \pm 6$ \\
\hline $\mathrm{DBP}[\mathrm{mm} \mathrm{Hg}]$ & $86 \pm 3$ & $84 \pm 4$ \\
\hline Heart rate $[\mathrm{bpm}]$ & $78 \pm 10$ & $74 \pm 14$ \\
\hline Total cholesterol [mmol/l] & $4.8 \pm 1.1$ & $4.9 \pm 1.3$ \\
\hline Triglyceride $[\mathrm{mmol} / \mathrm{l}]$ & $1.7 \pm 0.5$ & $1.8 \pm 0.6$ \\
\hline LDL-C [mmol/l] & $3.2 \pm 0.5$ & $3.1 \pm 0.6$ \\
\hline $\mathrm{HDL}-\mathrm{C}[\mathrm{mmol} / \mathrm{l}]$ & $1.2 \pm 0.5$ & $1.3 \pm 0.4$ \\
\hline $\mathrm{FPG}[\mathrm{mmol} / \mathrm{l}]$ & $5.9 \pm 0.3$ & $5.8 \pm 0.4$ \\
\hline Creatinine $[\mu \mathrm{mol} / \mathrm{l}]$ & $75.6 \pm 12.8$ & $72.1 \pm 15.6$ \\
\hline $\mathrm{ALB} / \mathrm{Cr}$ ratio $[\mu \mathrm{g} / \mathrm{mg}]$ & $28.3 \pm 13.2^{*}$ & $23.1 \pm 11.4$ \\
\hline IVS [mm] & $10.9 \pm 1.6^{*}$ & $10.0 \pm 1.3$ \\
\hline LVPW [mm] & $10.7 \pm 1.4^{*}$ & $9.9 \pm 1.6$ \\
\hline Left ventricular hypertrophy, $n$ (\%) & $10(27.0)^{\star}$ & $39(10.2)$ \\
\hline Albuminuria, $n(\%)$ & $8(21.6)^{\star}$ & $28(7.3)$ \\
\hline Dyslipidemia, $n$ (\%) & $5(13.5)$ & $47(12.3)$ \\
\hline Aspirin, $n(\%)$ & $3(8.1)$ & $30(7.8)$ \\
\hline Statins, $n(\%)$ & $3(8.1)$ & $42(11.0)$ \\
\hline cf-PWV $[\mathrm{m} / \mathrm{s}]$ & $10.6 \pm 0.4^{*}$ & $8.7 \pm 0.6$ \\
\hline
\end{tabular}

SBP-systolic blood pressure, DBP - diastolic blood pressure, LDL-C - low-density lipoprotein cholesterol, HDL-C - high-density lipoprotein cholesterol, FPG - fasting plasma glucose, ALB/Cr - albumin/creatinine, IVS - interventricular septal, LVPW - left ventricular posterior wall, cf-PWV - carotid-femoral pulse wave velocity, ${ }^{\star} P<0.05$ versus without arterial stiffness group.

\section{Risk factors for pre-hypertension}

Univariate and multivariate regression analysis was performed to evaluate potential risk factors for pre-hypertension. As presented in Table III, in the univariate regression analysis, aging, current cigarette smoking, increased fasting plasma glucose, presence of left ventricular hypertrophy, albuminuria and arterial stiffness were all significantly associated with pre-hypertension. In multivariate regression analysis, only aging and arterial stiffness remained significantly associated with pre-hypertension.

\section{Association between arterial stiffness and outcomes in prehypertension subjects}

To evaluate the association between cf-PWV and prevalence of left ventricular hypertrophy and albuminuria, logistic regression analysis was performed. As presented in Table IV, in the unadjusted model, increased cf-PWV was significantly associated with a 2.14-fold and 1.93-fold risk of left ventricular hypertrophy and albuminuria, respectively. With stepwise adjusted for potential covariates including age, male gender, fasting plasma glucose, presence of current cigarette smoking, dyslipidemia, statins and SBP, increased cf-PWV remained independently associated with left ventricular hypertrophy and albuminuria, with an increased odds of $41 \%$ and $24 \%$, respectively.

\section{Discussion}

The principal findings of our current study include four aspects. First, the prevalence of pre-hypertension in patients who came to the outpatient clinic for screening potential hypertension is $17.5 \%$ and the prevalence of target organ damage including left ventricular hypertrophy and albuminuria in subjects with pre-hypertension is not low. Second, compared to subjects without arteri- 
Table III. Logistic regression analysis of risk factors for pre-hypertension

\begin{tabular}{|lcc|}
\hline Parameter & \multicolumn{2}{c|}{ Pre-hypertension } \\
\cline { 2 - 3 } & $\begin{array}{c}\text { Univariate } \\
\text { OR }(95 \% \mathrm{Cl})\end{array}$ & $\begin{array}{c}\text { Multivariate } \\
\text { OR }(95 \% \mathrm{CI})\end{array}$ \\
\hline Age [years] & $1.32(1.18-1.49)$ & $1.06(1.02-1.14)$ \\
\hline Male gender & $1.07(0.91-1.10)$ & $1.04(0.91-1.12)$ \\
\hline Current cigarette smoking & $1.26(1.07-1.45)$ & $0.99(0.89-1.05)$ \\
\hline FPG [mmol/l] & $1.19(1.04-1.33)$ & $1.08(0.96-1.16)$ \\
\hline Creatinine $[\mu m o l / I]$ & $1.03(0.94-1.15)$ & $1.05(0.91-1.13)$ \\
\hline Left ventricular hypertrophy & $1.28(1.13-1.47)$ & \\
\hline Albuminuria & $1.20(1.10-1.33)$ & $1.28(1.14-1.36)$ \\
\hline Dyslipidemia & $1.08(0.95-1.16)$ & \\
\hline Arterial stiffness & $1.53(1.25-1.74)$ & \\
\hline
\end{tabular}

$F P G$ - fasting plasma glucose.

Table IV. Logistic regression analysis

\begin{tabular}{|lcc|}
\hline Model & $\begin{array}{c}\text { Left ventricular hypertrophy } \\
\text { OR }(95 \% \mathrm{Cl})\end{array}$ & $\begin{array}{c}\text { Albuminuria } \\
\text { OR }(95 \% \mathrm{Cl})\end{array}$ \\
\hline Unadjusted & $2.14(1.96-2.33)$ & $1.93(1.84-2.02)$ \\
\hline 1 & $1.90(1.76-2.11)$ & $1.74(1.56-1.93)$ \\
\hline 2 & $1.72(1.57-1.94)$ & $1.55(1.40-1.76)$ \\
\hline 3 & $1.41(1.25-1.57)$ & $1.24(1.13-1.40)$ \\
\hline
\end{tabular}

OR - odds ratio, Cl - confidence interval, Model 1 - adjusted for age and male gender, Model 2 - further adjusted for smoking status, fasting plasma glucose and dyslipidemia, Model 3 - further adjusted for statins and systolic blood pressure.

al stiffness, those with arterial stiffness are more likely to have left ventricular hypertrophy and albuminuria. Third, ageing and presence of arterial stiffness are two major potential risk factors for pre-hypertension. Fourth, in subjects with pre-hypertension, increased cf-PWV is associated with higher prevalence of target organ damage such as left ventricular hypertrophy and albuminuria.

Hypertension has become one of the most significant public health problems worldwide. Observational studies and meta-analysis further demonstrate that there is a linear relationship between SBP and target organ damage, cardiovascular diseases and all-cause mortality $[16,17]$. Therefore, from a long-term perspective, it is reasonable to speculate that management of pre-hypertension should be beneficial for reducing incident cardiovascular diseases and mortality. Moreover, prior observational studies also suggested that pre-hypertension is linked to high prevalence of comorbidities, indicating that subjects with pre-hypertension are a truly high-risk population $[18,19]$. Consistent with previous reports, the prevalence of target organ damage in subjects with pre-hypertension is also not low in our present study. Nevertheless, the prevalence of pre-hypertension in subjects who came to the outpatient clinic for screening hypertension was $17.5 \%$ in our outpatient clinic, which was much lower than in previous reports $[20,21]$. Subjects in our current research were relatively young and without overt cardiovascular and renal diseases. One might speculate that the differences in clinical characteristics between different studies might explain these discrepancies.

We further evaluated potential risk factors for pre-hypertension. Overall, we observed that ageing and presence of arterial stiffness were two independent risk factors for pre-hypertension. Notably, ageing is a major risk factor for BP elevation and the associated mechanisms are multifactorial. For example, ageing is significantly associated with glomerular filtration rate reduction, which in turn results in fluid retention and BP elevation. Notably, ageing is also a major risk factor for arterial stiffness [10]. However, in our current study, after adjustment for ageing, arterial stiffness remained significantly associated with pre-hypertension. From a pathophysiological perspective, on the one hand, reduced aortic distensibility leads to aortic 
pressure increase and SBP elevation [9]; on the other hand, the increased reflected wave from the peripheral artery contributes to aortic augmentation increase and BP elevation [22]. These pathophysiological changes might also be associated with left ventricular hypertrophy and albuminuria in subjects with arterial stiffness. Indeed, numerous observational studies indicate that arterial stiffness is an independent predictor for target organ damage and cardiovascular events in both general populations and hypertensive patients $[23,24]$.

Notably, target organ damage such as left ventricular hypertrophy and albuminuria is an important predictor for future cardiovascular and renal events in general hypertensive patients [25, 26]. Therefore, it is clinically relevant to manage potential reversible conditions so as to prevent target organ damage and improve cardiovascular prognosis. We observed that in pre-hypertensive subjects, the prevalence of left ventricular hypertrophy and albuminuria was not low, at $11.7 \%$ and $8.6 \%$, respectively. In the logistic regression model, after adjustment for potential covariates, we observed that arterial stiffness remained significantly associated with both left ventricular hypertrophy and albuminuria. Mechanistically, increased cf-PWV is associated with greater magnitude of blood flow back from the peripheral artery to the aortic artery during late systole, which in turn increases left ventricular afterload and causes left ventricular hypertrophy [27]. With respect to albuminuria, numerous prior observational studies indicate that albuminuria is significantly associated with arterial stiffness, particularly in subjects with hypertension and diabetes mellitus [28-30]. Importantly, extending previous findings, our current results show that in subjects with pre-hypertension, increased arterial stiffness was also associated with albuminuria. Based on the hemodynamic change related to arterial stiffness, one might speculate that in subjects with pre-hypertension, the change of renal glomerular pressure and infiltration rate might contribute to albuminuria [28].

There were several limitations of our current study. First of all, the inherent limitation of cross-sectional design could not allow us to confirm a causal relationship between arterial stiffness and left ventricular hypertension and albuminuria. However, findings from the current study provided novel insight into this relationship. Second, in spite of extensive adjustment for potential covariates, undetected and unmeasured residual covariates might still influence our findings. Third, subjects in our current study were relatively healthy and without overt cardiovascular and renal diseases; therefore, we should be aware that the findings might not be extrapolatable to other populations with different clinical characteristics. Nevertheless, our current study has two clinical implications. On the one hand, in subjects with pre-hypertension, in addition to measuring brachial BP, arterial stiffness evaluation could help to improve risk prediction of target organ damage. On the other hand, better understanding of potential cardiovascular risk could help physicians and patients to initiate primordial prevention, which in turn could reduce the cardiovascular burden in the future.

In conclusion, our preliminary findings suggest that in subjects with pre-hypertension, arterial stiffness is independently associated with left ventricular hypertrophy and albuminuria. The cfPWV may be a useful marker to identify who is at increased risk of target organ damage. Future randomized controlled trials are warranted to investigate whether improvement of arterial stiffness could prevent target organ damage in subjects with pre-hypertension.

\section{Acknowledgments}

Shaoyun Wu and Dubo Chen are co-first authors.

We thank Chen Zhang, PhD, for assisting us in performing statistical analysis of our paper.

The study was supported by grants from the Science and Technology project of Shenzhen city of China (JCYJ20170307161535847); and the study was supported by Sanming Project of Medicine in Shenzhen.

\section{Conflict of interest}

The authors declare no conflict of interest.

\section{References}

1. Ninomiya T, Perkovic $\mathrm{V}$, Turnbull $\mathrm{F}$, et al. Blood pressure lowering and major cardiovascular events in people with and without chronic kidney disease: meta-analysis of randomised controlled trials. BMJ 2013; 347: f5680.

2. Thomopoulos C, Parati G, Zanchetti A. Effects of blood pressure lowering on outcome incidence in hypertension: 3. Effects in patients at different levels of cardiovascular risk--overview and meta-analyses of randomized trials. J Hypertens 2014; 32: 2305-14.

3. Ghomari-Boukhatem H, Bouchouicha A, Mekki K, Chenni K, Belhadj M, Bouchenak M. Blood pressure, dyslipidemia and inflammatory factors are related to body mass index in scholar adolescents. Arch Med Sci 2017; 13: 46-52.

4. James PA, Oparil S, Carter BL, et al. 2014 evidence-based guideline for the management of high blood pressure in adults: report from the panel members appointed to the Eighth Joint National Committee (JNC 8). JAMA 2014; 311: 507-20.

5. Aronow WS. What should the target blood pressure goals be. Arch Med Sci 2016; 12: 1377-80. 
6. Mancia G, Fagard R, Narkiewicz K, et al. 2013 ESH/ESC Guidelines for the management of arterial hypertension: the Task Force for the management of arterial hypertension of the European Society of Hypertension $(E S H)$ and of the European Society of Cardiology (ESC). J Hypertens 2013; 31: 1281-357.

7. Lewington S, Clarke R, Qizilbash N, Peto R, Collins R. Age-specific relevance of usual blood pressure to vascular mortality: a meta-analysis of individual data for one million adults in 61 prospective studies. Lancet 2002; 360: 1903-13.

8. AlGhatrif $\mathrm{M}$, Strait JB, Morrell $\mathrm{CH}$, et al. Longitudinal trajectories of arterial stiffness and the role of blood pressure: the Baltimore Longitudinal Study of Aging. Hypertension 2013; 62: 934-41.

9. Kaess BM, Rong J, Larson MG, et al. Aortic stiffness, blood pressure progression, and incident hypertension. JAMA 2012; 308: 875-81.

10. Sun Z. Aging, arterial stiffness, and hypertension. Hypertension 2015; 65: 252-6.

11. Podgórski M, Grzelak P, Szymczyk K, Szymczyk E, Drożdż J, Stefańczyk L. Peripheral vascular stiffness, assessed with two-dimensional speckle tracking versus the degree of coronary artery calcification, evaluated by tomographic coronary artery calcification index. Arch Med Sci 2015; 11: 122-9.

12. Chobanian AV, Bakris GL, Black HR, et al. The Seventh Report of the Joint National Committee on Prevention, Detection, Evaluation, and Treatment of High Blood Pressure: the JNC 7 report. JAMA 2003; 289: 2560-72.

13. Côté AM, Brown MA, Lam E, et al. Diagnostic accuracy of urinary spot protein : creatinine ratio for proteinuria in hypertensive pregnant women: systematic review. BMJ 2008; 336: 1003-6.

14. Laurent S, Cockcroft J, Van Bortel L, et al. Expert consensus document on arterial stiffness: methodological issues and clinical applications. Eur Heart J 2006; 27 2588-605.

15. Cheitlin MD, Armstrong WF, Aurigemma GP, et al. ACC/AHA/ASE 2003 Guideline Update for the Clinical Application of Echocardiography: summary article. A report of the American College of Cardiology/American Heart Association Task Force on Practice Guidelines (ACC/AHA/ASE Committee to Update the 1997 Guidelines for the Clinical Application of Echocardiography). J Am Soc Echocardiogr 2003; 16: 1091-110.

16. Hsia J, Margolis KL, Eaton CB, et al. Prehypertension and cardiovascular disease risk in the Women's Health Initiative. Circulation 2007; 115: 855-60.

17. Liszka HA, Mainous AG, King DE, Everett CJ, Egan BM. Prehypertension and cardiovascular morbidity. Ann Fam Med 2005; 3: 294-9.

18. Greenlund KJ, Croft JB, Mensah GA. Prevalence of heart disease and stroke risk factors in persons with prehypertension in the United States, 1999-2000. Arch Intern Med 2004 164: 2113-8.

19. Zhang Y, Lee ET, Devereux RB, et al. Prehypertension, diabetes, and cardiovascular disease risk in a population-based sample: the Strong Heart Study. Hypertension 2006; 47: 410-4.

20. Khanam MA, Lindeboom W, Razzaque A, Niessen L, Milton $\mathrm{AH}$. Prevalence and determinants of pre-hypertension and hypertension among the adults in rural Bangladesh: findings from a community-based study. BMC Public Health 2015; 15: 203.

21. Wang Y, Wang QJ. The prevalence of prehypertension and hypertension among US adults according to the new joint national committee guidelines: new chal lenges of the old problem. Arch Intern Med 2004; 164 2126-34.

22. Van Bortel LM, Struijker-Boudier HA, Safar ME. Pulse pressure, arterial stiffness, and drug treatment of hypertension. Hypertension 2001; 38: 914-21.

23. Ben-Shlomo Y, Spears M, Boustred C, et al. Aortic pulse wave velocity improves cardiovascular event prediction: an individual participant meta-analysis of prospective observational data from 17,635 subjects. J Am Coll Cardiol 2014; 63: 636-46.

24. Mitchell GF, Hwang SJ, Vasan RS, et al. Arterial stiffness and cardiovascular events: the Framingham Heart Study. Circulation 2010; 121: 505-11.

25. Cha RH, Kim S, Ae YS, et al. Association between blood pressure and target organ damage in patients with chronic kidney disease and hypertension: results of the APrODiTe study. Hypertens Res 2014; 37: 172-8.

26. Zakopoulos N, Manios E, Moulopoulos S. Guidelines for the management of hypertension and target organ damage. J Hypertens 2013; 31: 2463-4.

27. Jia CF, Wang ZQ, Sun XX, Yang ZQ, Zou YJ, Jiang YN. Ascending aortic distensibility and target organ damage in primary hypertension without diabetes. Int J Cardiovasc Imaging 2017 Mar 16. doi: 10.1007/s10554-0171099-x. [Epub ahead of print]

28. Liu CS, Pi-Sunyer FX, $\mathrm{Li} \mathrm{Cl}$, et al. Albuminuria is strongly associated with arterial stiffness, especially in diabetic or hypertensive subjects - a population-based study (Taichung Community Health Study, TCHS). Atherosclerosis 2010; 211: 315-21.

29. Bouzas-Mosquera A, Peteiro J, Alvarez-García N. Exercise blood pressure response, albuminuria, and arteria stiffness in patients with hypertension. Am J Med 2009; 122: e9; author reply e11.

30. Bouchi R, Babazono T, Mugishima M, et al. Arterial stiffness is associated with incident albuminuria and decreased glomerular filtration rate in type 2 diabetic patients. Diabetes Care 2011; 34: 2570-5. 